\title{
Subcellular object quantification with Squassh3C and SquasshAnalyst
}

\author{
Aurélien Rizk ${ }^{\dagger}$, Maysam Mansouri, Kurt Ballmer-Hofer, and \\ Philipp Berger \\ Paul Scherrer Institute, Biomolecular Research, Molecular Cell Biology, \\ Villigen, Switzerland
}

† Present affiliation: Algorizk SARL, France

BioTechniques 59:309-312 (November 2015) doi 10.2144/000114352

Keywords: image analysis; segmentation; co-localization; live-cell imaging

Supplementary material for this article is available at www.BioTechniques.com/article/114352.

Quantitative image analysis plays an important role in contemporary biomedical research. Squassh is a method for automatic detection, segmentation, and quantification of subcellular structures and analysis of their colocalization. Here we present the applications Squassh3C and SquasshAnalyst. Squassh3C extends the functionality of Squassh to three fluorescence channels and live-cell movie analysis. SquasshAnalyst is an interactive web interface for the analysis of Squassh3C object data. It provides segmentation image overview and data exploration, figure generation, object and image filtering, and a statistical significance test in an easy-to-use interface. The overall procedure combines the Squassh3C plug-in for the free biological image processing program Image $J$ and a web application working in conjunction with the free statistical environment R, and it is compatible with Linux, MacOS X, or Microsoft Windows. Squassh3C and SquasshAnalyst are available for download at www.psi.ch/lbr/SquasshAnalystEN/SquasshAnalyst.zip.

Many areas of biomedical research rely on cell imaging to obtain information about the phenotypic response of cells to different growth factors and drugs. A common task is the quantification of spatial colocalization between fluorescently labeled species since the proteins under study are usually related to known subcellular markers.

Squassh is a subcellular shape analysis tool based on a segmentation technique that corrects for microscope blur, detector noise, and uneven background $(1,2)$. It enables the generation of large amounts of data on the features of subcellular objects. Once the objects of interest are distinguished from the background, object features such as size, intensity, or shape, as well as colocalization between fluorescence channels, are calculated and then written to comma-separated value (CSV) files.
Squassh comes with basic $R$ functionalities for data analysis, figure generation, and statistical tests. However, the investigation of data generated for a particular problem often requires customized analysis, which then requires learning the $\mathrm{R}$ programming language, a prohibitive investment of time for many cellular biology researchers who lack training in software programming. Moreover, analysis of more than two fluorescence channels or of live-cell imaging is even more complex since specific data processing steps are necessary.

Here we present an updated version of Squassh called Squassh3C that works in combination with SquasshAnalyst, a new interactive web program written in the statistical programming environment R. This allows for the analysis of images (single images or movies obtained from fixed- or live-cell imaging) having up to three fluorescence channels that can be obtained, for example, with the co-expression of fluorescently tagged sensors (3) or with immunostaining. $R$ is a free software and programming language (www.r-project.org) providing a wide variety of data processing, statistical, and graphical tools. SquasshAnalyst brings these tools to non-programmers by providing an easy-to-use graphical interface made with the R package Shiny (www.rstudio.com/shiny).

The workflow functions with image data files in any format supported by ImageJ. In contrast to Squassh, Squassh3C can analyze in batch mode different experimental conditions separated into distinct subfolders by selecting the parent folder. Conditions can then be compared and tested for statistical significance in the SquasshAnalyst user interface. It is also possible to compare conditions from separate Squassh3C computational runs.

Squassh3C is implemented as a plugin for the image processing program ImageJ $(4,5)$. It provides a user interface for input selection, segmentation parameters, setting the microscope objective characteristics, and output options (Figure 1A). SquasshAnalyst is a web application with user interface tabs

\section{METHOD SUMMARY}

Here we describe the image analysis applications Squassh3C, a method for automatic segmentation and quantification of subcellular structures and analysis of their co-localization in up to three fluorescence channels, and SquasshAnalyst, an interactive web interface for the analysis of Squassh3C segmentation data. 

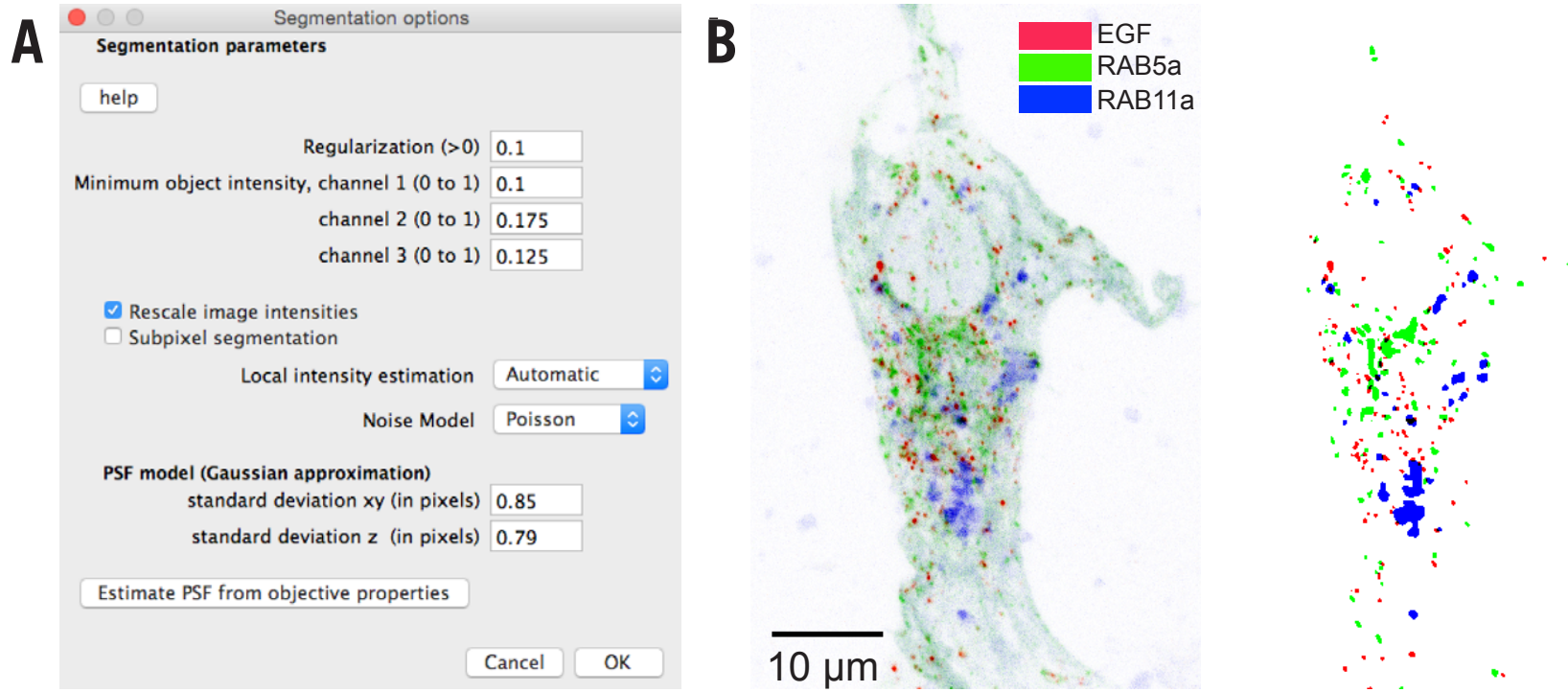

Figure 1. Squassh 3C. (A) Squassh3C interface showing segmentation parameters panel. A higher regularization parameter should be used for noisy images. Minimum object intensities are set separately for each channel. Further description of the parameters is available by clicking on the help button. (B) A COS cell transiently expressing EYFP-RAB5a (green) and mCherry-RAB11a (blue) is shown 30 min after stimulation with EGF-Cy5 (red). A z-axis projection of the fluorescence image is shown to the left of the Squassh segmentation (right). Lookup table values ranging from white (background) to color (maximum signal) produce a printer friendly colormap.

A

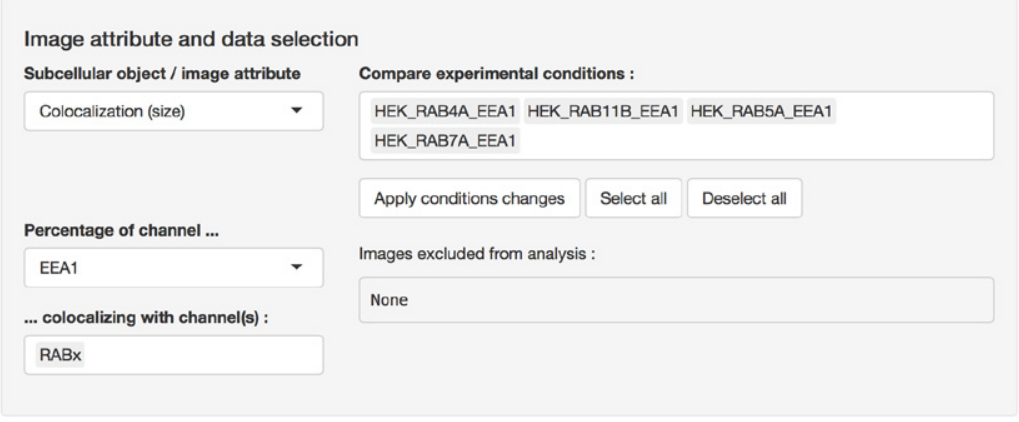

\section{Chart type}

$$
\text { Strip chart }
$$
$+$

Sort conditions

LExport pdf

¿ Export csv
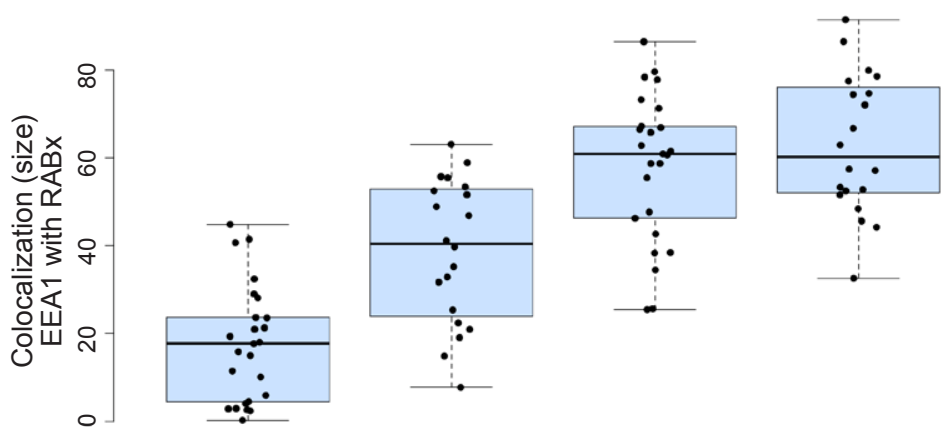

HEK RAB7A EEA1 HEK_RAB11B_EEA1 HEK_RAB5A_EEA1 HEK_RAB4A_EEA 1
B

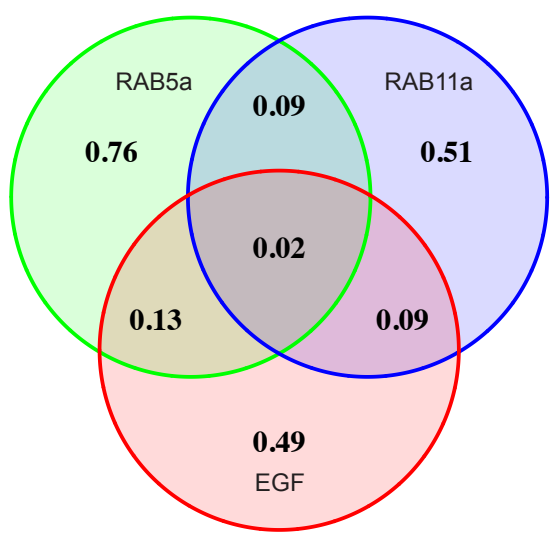

C

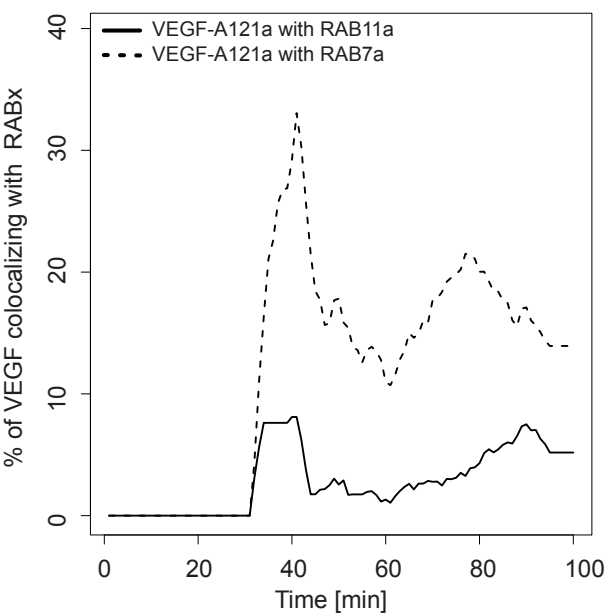

Figure 2. SquasshAnalyst. (A) SquasshAnalyst interface showing analysis tab with settings for a comparison of the percentages of early endosome marker EEA1 colocalizing with RAB7a, RAB11b, RAB5a, and RAB4a. (B) Venn diagram showing the average colocalization of five fluorescence images containing three fluorescence channels: RAB5a (green), RAB11a (blue), and EGF (red). (C) PAE cells expressing VEGFR-2 and NRP-1 receptors were transiently infected with a virus expressing mTFP1-RAB7a and mCherry-RAB11a and then stimulated with Cy5-labeled VEGF165a. The graph displays the change over time of the amount of VEGF colocalizing with RAB7a or RAB11a. We provide all microscopy images needed to reproduce these figures with SquasshAnalyst at tinyurl.com/squasshanalystdata. 
for data input selection, an overview of Squassh3C generated segmentation, and data analysis (Figure 2A). Figures generated by SquasshAnalyst can be live-previewed while their graphical, processing, and analysis options are tuned, and can then be exported as PDF or PNG files.

Squassh3C and SquasshAnalyst form an automated workflow for the analysis of up to thousands of fluorescence images. However, for correct analysis, it is important to check that the intermediate steps, such as segmentation of individual images or filtering of outliers, are done properly. SquasshAnalyst therefore generates a contact sheet view containing thumbnails of the segmented images to facilitate inspection of Squassh3C segmentation analysis. Each thumbnail displays the original image and the segmented objects side-byside. The original image is displayed with all channels merged, automatically enhanced contrast with $0.05 \%$ of the pixels saturated, and maximum z-axis projection in the case of 3-D images (Figure 1B). When a cell mask of positively transfected cells is computed by Squassh3C, the outline of the mask is overlaid in white on the image. It is possible from the contact sheet view to exclude selected images from further analysis.

The visual comparison of an image feature (colocalization, object count, cell size, mean object volume, mean object intensity, mean object sphericity) between image sets is achieved with bar plots, boxplots, or strip charts. Figure 2A compares colocalization of the EEA1 marker for early endosomes with four different RAB GTPases. The lesser colocalization of EEA1 with RAB11b and RAB7a compared with RAB4a and RAB5a reflects the maturation of early endosomes to recycling and degradation vesicles, respectively. Colocalization quantified after segmentation as done with Squassh3C was shown to yield better results compared with pixel-based approaches such as Pearson correlation (1,2).
An option allows switching between colocalization-based on object count, overall overlap size, or overall signal colocalization.

Fast visualization of all colocalization channel subsets in a given image set is achieved by displaying them as a Venn diagram (Figure 2B).

Quantitative analysis of movies from live-cell imaging is done by separate analysis of the movie frames. Results are then combined to display the progression over time of the colocalization of selected channel subsets or of any mean image feature. A rolling average over time frames with a parameterizable time window can be used to smooth any noise. As an example, we quantified the localization of VEGF165a-Cy5 with mTFP1-RAB7a and mCherry-RAB11a in PAE cells stably expressing VEGFR-2 and NRP-1 receptors (Figure $2 \mathrm{C}$ ) in order to monitor, respectively, the degradation and recycling of VEGF165a after VEFGR2 stimulation. (See Reference 6 for a description of the results.)

Statistical analysis to compare multiple conditions at a time and for pairwise multiple comparisons are done, respectively, with one-way ANOVA and Tukey-Kramer tests or with the nonparametric Kruskal-Wallis and Dunn tests. The most representative image of a condition is automatically detected and displayed. It is possible to remove objects from the analysis based on intensity or shape criteria thresholds. Removing objects with low intensity from the analysis is useful, for instance, to remove non-specific fluorescence in immunostaining experiments.

Squassh3C and SquasshAnalyst constitute a complete workflow from segmentation to data analysis that is based on the state-of-the-art segmentation method Squassh (1,2). A tutorial and installation instructions for the workflow are available in the SquasshAnalyst manual at www.psi. ch/lbr/SquasshAnalystEN/SquasshAnalyst_ manual.pdf. An included toy example allows

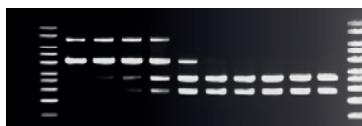

\section{Minicircle \& Plasmid DNA Service}

QC including CGE service for topology analysis

- Stability and storage studies

InStock service for reporter gene plasmids and minicircles (GFP, lacZ, luc, miniS/MAR etc.)

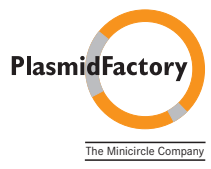

店 testing of the entire procedure in less than 15 minutes. Finally, the manual showcases further possible uses of SquasshAnalyst with the image data sets corresponding to Figure 2 containing more than 300 images in total.

\section{Author contributions}

P.B., K.B., and A.R. designed the project; A.R. developed the image-processing algorithm and programmed the software with input from P.B., K.B., and M.M.; experimental data were obtained by M.M.; A.R. and P.B. wrote the manuscript with input from K.B. and M.M; figures were prepared by A.R. and P.B.

\section{Acknowledgments}

The research leading to these results has received funding from the European Community's Seventh Framework Programme (FP7/2007-2013) under grant agreement no. 290605 (PSI-FELLOW/COFUND).

\section{Competing interests}

The authors declare no competing interests.

\section{References}

1. Rizk, A., G. Paul, P. Incardona, M. Bugarski, M. Mansouri, A. Niemann, U. Ziegler, P. Berger, and I.F. Sbalzarini. 2014. Segmentation and quantification of subcellular structures in fluorescence microscopy images using Squassh. Nat. Protoc. 9:586-596.

2. Paul, G., J. Cardinale, and I.F. Sbalzarini. 2013. Coupling Image Restoration and Segmentation: A Generalized Linear Model/ Bregman Perspective. Int. J. Comput. Vis. 104:69-93.

3. Kriz, A., K. Schmid, N. Baumgartner, U. Ziegler, I. Berger, K. Ballmer-Hofer, and P. Berger. 2010. A plasmid-based multigene expression system for mammalian cells. Nat Commun. 1:120.

4. Collins, T.J. 2007. ImageJ for microscopy. Biotechniques. 43:25-30.

5. Schneider, C.A., W.S. Rasband, and K.W Eliceiri. 2012. NIH Image to ImageJ: 25 years of image analysis. Nat. Methods 9:671-675.

6. Ballmer-Hofer, K., A.E. Andersson, L.E. Ratcliffe, and P. Berger. 2011. Neuropilin-1 promotes VEGFR-2 trafficking through Rab11 vesicles thereby specifying signal output. Blood 118:816-826

Received 15 June 2015; accepted 05 August 2015.

Address correspondence to Philipp Berger, Paul Scherrer Institute, Molecular Cell Biology, Villigen, Switzerland. E-mail: Philipp.Berger@psi.ch

To purchase reprints of this article, contact: biotechniques@fosterprinting.com 